\title{
Kernos
}

Revue internationale et pluridisciplinaire de religion grecque antique

33 | 2020

Varia

\section{La vie religieuse à Paphos à travers les sources épigraphiques : acteurs, espaces, nouvelles perspectives}

\section{Stefano Caneva}

\section{(2) OpenEdition}

1 Journals

\section{Édition électronique}

URL : https://journals.openedition.org/kernos/3479

DOI : $10.4000 /$ kernos.3479

ISSN : 2034-7871

\section{Éditeur}

Centre international d'étude de la religion grecque antique

\section{Édition imprimée}

Date de publication : 31 décembre 2020

Pagination : 307-315

ISBN : 978-2-87562-264-8

ISSN : 0776-3824

\section{Référence électronique}

Stefano Caneva, "La vie religieuse à Paphos à travers les sources épigraphiques : acteurs, espaces, nouvelles perspectives », Kernos [En ligne], 33 | 2020, mis en ligne le 31 décembre 2020, consulté le 06 décembre 2022. URL : http://journals.openedition.org/kernos/3479; DOI : https://doi.org/10.4000/ kernos.3479

Ce document a été généré automatiquement le 6 décembre 2022.

Tous droits réservés 


\title{
La vie religieuse à Paphos à travers les sources épigraphiques : acteurs, espaces, nouvelles perspectives
}

\author{
Stefano Caneva
}

\section{RÉFÉRENCE}

Jean-Baptiste CAYLA, Les Inscriptions de Paphos. La cité chypriote sous la domination lagide et à l'époque impériale, Lyon, Mom Éditions, 2018. 1 vol. $21 \times 30 \mathrm{~cm}, 565$ p. (Travaux de la Maison de l'Orient et de la Méditerranée, 74). ISBN : 978-2-35668-056-3.

1 Cet article critique analyse le volume que Jean-Baptiste Cayla a récemment consacré aux inscriptions grecques alphabétiques de Paphos, aux périodes hellénistique et impériale ${ }^{1}$. Le livre constitue la publication, longtemps attendue, de la thèse de doctorat soutenue par l'auteur en 2003 sur le dossier des inscriptions alphabétiques grecques et latines de la région de Paphos (sanctuaire de Palaipaphos, ville de Nea Paphos et chōra, y compris l'îlot de Géronisos). Dans l'avant-propos, l'A. explique que le travail de terrain date d'avant 2003, bien que d'importantes mises à jour bibliographiques aient été accomplies lors de la préparation de ce volume. Le dossier épigraphique se compose de 384 entrées, pour la plupart en grec. On compte aussi deux inscriptions digraphes en syllabique chypriote et grec alphabétique ( $\left.{ }^{\circ s} 1,223\right)$, sept inscriptions bilingues gréco-latines et 20 inscriptions latines. Les documents inédits publiés ici sont au nombre de seize et, pour quatre autres inscriptions, on ne dispose que de la description de la pierre, l'A. n'ayant pas obtenu d'autorisation de publication (cf. p. 469-470 pour un catalogue des textes). L'édition des documents est complétée par une introduction sur la topographie de la région de Paphos, l'histoire des études et les types de supports ; un aperçu historique, à la fois diachronique et thématique ; une bibliographie; plusieurs tables des matières; une table des concordances; un riche apparat photographique de 280 images. 
2 Le dossier épigraphique est divisé entre périodes hellénistique et impériale, et il est organisé en sections thématiques. Il offre un cadre varié de la vie politique, sociale et culturelle de la région de Paphos entre la fin du $\mathrm{IV}^{\mathrm{e}} \mathrm{s}$. av. J.-C. et le IV $\mathrm{e}$ s. ap. J.-C. environ. Dans le présent propos, on se concentrera sur les documents ayant un intérêt pour l'histoire religieuse et culturelle. On discutera plus particulièrement les textes pour lesquels l'édition de Cayla apporte des améliorations significatives à notre compréhension ou, au contraire, soulève des problèmes qui n'ont pas reçu une solution satisfaisante dans le présent volume.

\section{Cultes, honneurs et administration à Paphos : la perspective du sanctuaire d'Aphrodite}

Dans la première section, concernant les documents du règne de Nikoklès, la base d'une statue dédiée par le même roi $\left(n^{\circ} 3\right)$, peut-être à la suite d'un oracle de la déesse, témoigne pour la première fois de l'utilisation de l'épiclèse Paphia pour la Wanassa de Paphos. Le corpus hellénistique est largement dominé par les documents concernant, de manière directe ou indirecte, les Ptolémées. Le dossier révèle l'importance du sanctuaire d'Aphrodite en tant que lieu de mise en place de la hiérarchie de la société de Paphos, dans laquelle les soldats et les gouverneurs jouent un rôle fondamental. Cette dynamique se manifeste notamment à travers la dédicace de statues honorifiques: aucun sanctuaire chypriote n'a préservé autant de bases de statues honorifiques que celui d'Aphrodite à Palaipaphos à la période hellénistique. Cayla offre une synthèse utile des changements diachroniques dans le formulaire des dédicaces à Aphrodite, se focalisant en particulier sur la chronologie de l'introduction de la dénomination Aphrodite Paphia ainsi que sur la position qu'elle occupe dans les textes inscrits sur les bases de statues (p. 93-94). L'A. souligne aussi le silence des institutions de la cité dans cette logique honorifique, ce qui révèle son état de ville sujette aux initiatives de l'élite lagide. Les documents recueillis permettent d'étudier l'entrelacement entre l'administration de Chypre et le développement du culte royal entre le $\mathrm{III}^{\mathrm{e}}$ et le $\mathrm{I}^{\mathrm{er}} \mathrm{s}$. av. J.-C., en révélant un changement significatif de la distribution sociale des cultes pour les Ptolémées. La haute période hellénistique est caractérisée par la grande diffusion des dédicaces de qualité modeste, et pour la plupart anonymes, composant le dossier d'Arsinoé II ( $\left.{ }^{\text {os }} 4-10\right)$. Au II ${ }^{\text {e }}$ s., en revanche, l'initiative dans le soutien au culte royal passe aux plus hauts représentants de l'administration et de la hiérarchie de cour lagide, selon une tendance attestée partout dans le royaume ptolémaïque ${ }^{2}$. C'est l'époque où les gouverneurs (stratēgoi) de Chypre servent aussi comme archiereis des cultes sur l'île et assument souvent la fonction de prêtres à vie du culte des membres de la maison royale. Dans les documents de la région de Paphos, c'est le cas d'Hélénos, prêtre à vie de Cléopâtre (III) Thea Aphrodite Euergetis, selon la nouvelle restitution du texte proposée par Cayla $\left(\mathrm{n}^{\circ} 20\right)^{3}$, et de Séleukos, prêtre à vie ${ }^{4} \mathrm{de}$ Cléopâtre II

ou $\operatorname{III}^{5}\left(\mathrm{n}^{\circ} 44\right)$. Du règne de Ptolémée IX (88-80 av. J.-C.) date la dédicace à Aphrodite Paphia, par la cité de Paphos, d'une statue d'Onésandros, dont les charges révèlent une fonction charnière entre l'administration locale et la cour d'Alexandrie $\left(n^{\circ} 89\right)$ : Onésandros est « parent du roi, prêtre à vie du roi Ptolémée Theos Sôter ainsi que du Ptolémaion qu'il a fondé, secrétaire de la cité de Paphos et chef de la grande bibliothèque à Alexandrie ». Le financement d'un sanctuaire du roi par la personne qui 
exerce la fonction de prêtre du même souverain illustre l'importance du culte royal dans les stratégies d'autopromotion des élites au niveau local ainsi qu'à l'échelle du royaume $^{6}$. Au-delà d'une mise à jour des mécanismes de l'administration, le rôle promoteur des élites dans la diffusion des cultes pour les souverains apparait comme un facteur de continuité, non seulement à la période hellénistique, mais aussi, comme on le verra, à l'époque impériale. À ce propos, les témoignages de la vie des mercenaires égyptiens à Paphos sont aussi d'un grand intérêt. Même si la garnison était stationnée à Nea Paphos, le sanctuaire de Palaipaphos occupait une place prééminente dans la publication de documents associés à la vie des soldats (voir par exemple la liste de contributeurs pour l'approvisionnement de l'huile pour le gymnase, $\mathrm{n}^{\circ} 66$ ). Le document fragmentaire $\mathrm{n}^{\circ} 70$, contenant une pétition des soldats à Ptolémée VI ou VIII,

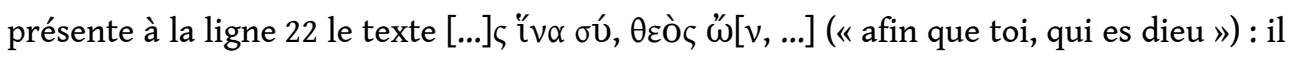
s'agit d'une formule par laquelle les auteurs de la pétition font appel à un langage divinisant pour exprimer une captatio benevolentiae envers le souverain. Il faut remarquer que ce texte témoigne d'une utilisation rhétorique du vocabulaire des cultes aux souverains, bien avant les cas parallèles que nous font connaitre quelques documents provenant d'Alexandrie et de l'Égypte entre la fin de la période hellénistique et le début du Principat. Ces attestations plus tardives se laissent donc interpréter comme l'extension, à des contextes de communication plus larges, d'un usage développé originairement dans le cadre des relations entre des membres de l'armée ou de l'administration lagide et les souverains? ${ }^{7}$.

\section{Une nouvelle chronologie pour les « technites de Dionysos et des Theoi Euergetai »}

Les documents concernant l'association des technites dionysiaques ( $\left.\mathrm{n}^{\text {os }} 90-96\right)$ sont ceux pour lesquels Cayla apporte la contribution la plus innovante à l'histoire politique et culturelle de Chypre. L'A. montre de manière convaincante que certains de ces textes, notamment ceux où l'association porte le nom de « technites de Dionysos et des Theoi Euergetai " ( $\mathrm{n}^{\text {os }}$ 93-95) ne peuvent dater de la fin du II ${ }^{\mathrm{e}}$ s., comme le soutient la communis opinio, mais remontent à la période de la "restauration lagide " à Chypre entre 48 av. J.-C. (date de la restitution de l'île à Cléopâtre par César) et 31 av. J.-C. Le confirment les traits d'écriture tardifs ainsi que l'absence de tout titre aulique attaché aux personnages honorés dans ces textes. Ce détail ne se justifie que par rapport au déclin et à la disparition du système de titulature de cour lagide au cours du $\mathrm{I}^{\mathrm{er}} \mathrm{s}$. Cette nouvelle datation indique un changement ultérieur des acteurs impliqués dans le culte royal, qui s'identifient désormais à une association professionnelle directement liée à l'élite paphienne. De plus ${ }^{8}$, la dénomination d'Euergetai ne peut guère renvoyer au couple de Ptolémée VIII et Cléopâtre III, comme le pensaient les chercheurs précédents, mais bien à Cléopâtre VII et Antoine, ce qui invite à réduire la fourchette chronologique aux années entre 41 (après la rencontre de Tarse) et 31 av. J.-C. Dans le cadre d'une expérimentation cultuelle et dynastique d'avant la guerre contre Octavien, la nouvelle visibilité des Technites dans le sanctuaire d'Aphrodite à Palaipaphos s'explique bien : le lien entre Dionysos, dieu de l'association, et la déesse de Paphos reflète les tendances contemporaines de l'idéologie d'Alexandrie, et en particulier le message de l'union entre Antoine/Dionysos et Cléopâtre/Aphrodite à la suite de la rencontre de Tarse de 41 av. J.-C. Pour finir, la nouvelle datation des documents concernant les technites 
implique aussi la mise à jour d'une autre question majeure dans l'histoire de Chypre : l'institution du Koinon de Chypre, mentionné dans la dédicace à Aphrodite Paphia n ${ }^{\circ}$ 93, apparaît désormais comme une innovation à dater de la brève domination romaine (58$48 \mathrm{av}$. J.-C.) et non comme l'étape terminale de l'unification de l'île sous les Ptolémées à la fin du $\mathrm{II}^{\mathrm{e}} \mathrm{s}$.

\section{L'interprétation du culte royal ptolémaïque : quelques mises au point nécessaires}

5 À côté de ces mérites dans la compréhension des changements politiques et culturels de la Paphos hellénistique, on regrette toutefois que, pour d'autres questions, Cayla ne fasse que re-proposer des solutions datées et déjà écartées par la critique. C'est probablement une conséquence d'un manque de mise à jour de la bibliographie à ce propos, au moment de la préparation du volume. Par exemple, en discutant le dossier des petites dédicaces pour Arsinoé Philadelphos, Cayla suit la thèse de Louis Robert ${ }^{9}$, selon laquelle les autels portatifs et les petites plaques dédiés à Arsinoé seraient utilisés lors des processions civiques pour la reine divinisée afin de permettre aux citoyens de participer à l'évènement public par des offrandes personnelles et familiales devant leurs maisons. Or cette interprétation fut développée à partir du fragment d'un règlement rituel d'Alexandrie cité par l'historien Satyrus (P.Oxy. XXVII 2465, fr. 2). Elle explique bien le fonctionnement de la procession d'Arsinoé dans la capitale lagide, qui fait l'objet du règlement sacré cité dans ce texte. Pourtant, il faudrait éviter de généraliser ce point de vue au détriment de la variété de la documentation concernant le culte de cette reine divinisée à travers l'empire lagide ${ }^{10}$. Ainsi, dans l'aperçu historique (p. 70-71), Cayla observe avec raison que les dédicaces paphiennes renvoient dans la plupart des cas au contexte du sanctuaire d'Aphrodite plutôt qu'à un cadre domestique (qui, à Paphos, n'est jamais attesté de manière certaine), et que les supports appartiennent à des typologies différentes. Néanmoins, dans l'édition (p. 128-130), l'A. revient sur une position plus conservatrice, décrivant comme autels tous les objets, y compris ceux qui ne peuvent clairement pas en être. La thèse selon laquelle le culte d'Arsinoé à Paphos commença durant la vie de la reine ${ }^{11}$ et que le culte paphien joua un rôle décisif dans la définition du culte d'Arsinoé à Alexandrie est également à rejeter. Avancée par Młyniarczyk, qui voyait dans un culte d'Aphrodite sur le promontoire paphien de Zéphyria le précédent et le modèle de la caractérisation marine d'Arsinoé (et peut-être même du transfert, d'ailleurs non prouvé, du toponyme Zéphyrion près d'Alexandrie), cette hypothèse avait déjà été rejetée par Anastassiades et n'est plus retenue par les spécialistes ${ }^{12}$. Une autre hypothèse complémentaire est aussi sans fondement: celle qui identifie le Boïskos, père de la prêtresse paphienne Phanion $\left(n^{\circ} 80\right)$, au père de l'amiral lagide Kallikratès (honoré dans le texte $\left.\mathrm{n}^{\circ} 65\right)$ et en déduit la provenance chypriote du personnage. De fait, rien ne permet de contester l'origine samienne de Kallikratès, qui est bien attestée dans les documents : le Boïskos père de Phanion et le père de l'amiral et fondateur du sanctuaire d'Arsinoé au Cap Zéphyrion ne sont donc que des homonymes. S'il y a certainement un lien entre le culte de la reine divinisée dans les régions d'Alexandrie et de Paphos, on pourrait plutôt penser à une influence en sens inverse, depuis la capitale ptolémaïque dans le Delta jusqu'à la capitale provinciale à Chypre. 
Une autre problématique mérite un réexamen critique. En discutant le profil social des titulaires des prêtrises à vie pour le culte royal ptolémaïque à Chypre aux $\mathrm{II}^{\mathrm{e}}$ et $\mathrm{I}^{\mathrm{er}} \mathrm{s}$. av. J.-C., Cayla attire l'attention sur leur genre, qui est toujours masculin. Ce détail constitue une différence importante par rapport aux prêtrises connues en Égypte et l'A. voit dans cette particularité le signe d'une continuité avec la tradition paphienne des rois Kinyridai, qui étaient aussi prêtres de la Wanassa à Palaipaphos. Bien qu'elle soit fascinante, cette hypothèse surestime l'enracinement local d'une tendance qui est en fait bien plus répandue. À cet égard, nous pouvons comparer le dossier de Palaipaphos avec l'inscription préservée sur un fragment de colonne provenant d'un gymnase chypriote non localisé, à dater sur base paléographique $d u \mathrm{II}^{\mathrm{e}} \mathrm{s}$. av. J.-C. Ici, nous apprenons que le gymnasiarque servait également comme prêtre d'Arsinoé Philadelphos. Nous ne savons pas si cette tâche était aussi viagère, mais nous percevons en tout cas une situation très différente de celle $\mathrm{du}_{\mathrm{III}}{ }^{\mathrm{e}} \mathrm{s}$., lorsqu'un document phénicien d'Idalion nous fait connaître une kanephoros d'Arsinoé, évidemment modelée sur la prêtrise homonyme d'Alexandrie ${ }^{13}$. De ce point en dérive un autre. Cayla (p. 112-113) voit dans l'association entre reines lagides et prêtres masculins à Chypre un trait proprement paphien du culte royal lagide et un signe de continuité entre le lien Arsinoé II - Kallikratès au $\mathrm{III}^{\mathrm{e}} \mathrm{s}$. d'une part, et celui entre les reines du $\mathrm{II}^{\mathrm{e}} \mathrm{s}$. et les gouverneurs archiereis d'autre part. Il me semble que cette interprétation va au-delà de ce que nous pouvons lire dans les documents, et cela non seulement parce que, comme on a vu, l'hypothèse d'une origine chypriote de Kallikratès est à écarter, mais aussi en considération du fait que nos sources ne permettent pas d'identifier un lien explicite entre les reines lagides $d u \mathrm{II}^{\mathrm{e}}$ s., les gouverneurs, et la protection de la navigation. On notera à ce propos que le titre de navarque n'est porté, dans les inscriptions de Paphos, que par Kallikratès $\left(n^{\circ} 65\right)$ : un lien explicite avec la mer n'est donc pas un trait caractérisant le profil des gouverneurs de Chypre au II $^{\mathrm{e}} \mathbf{s}$. L'absence de prêtresses dans le culte royal paphien de l'époque ne s'explique pas sur le plan religieux, mais dépend plutôt du fait qu'en qualité d'archiereis, les gouverneurs de l'île étaient responsables du culte royal dans sa totalité.

On peut conclure cette section avec une précision chronologique à propos du texte $\mathrm{n}^{\circ} 13$, conservé sur la base d'une statue de Ptolémée V Theos Épiphanès et Eucharistos érigée par le stratège Polykratès. Puisque Polykratès fut gouverneur entre 203 et 197, et que Cayla considère qu'il est probable que Ptolémée V n'a pas porté son titre de Theos Épiphanès (parfois suivi d'Eucharistos) avant sa majorité (il est né en octobre 210), l'A. se trouve forcé d'avancer l'hypothèse que Polykratès a utilisé cette titulature de son initiative, avant qu'elle ne devienne officielle. Cette interprétation se heurte au constat que, dans les dédicaces aux souverains accomplies par des membres de l'élite, les auteurs n'apportaient généralement pas d'innovations concernant les dénominations utilisées, mais suivaient plutôt un protocole royal établi et bien reconnaissable ${ }^{14}$. La solution au problème posé par ce texte vient plutôt d'une étude récente de Lanciers, qui a montré que le culte alexandrin de Ptolémée $V$ avait déjà débuté en 199, lorsque le roi avait seulement 11 ans. Le double titre d'Épiphanès et Eucharistos, moins commun et apparemment moins officiel que la simple épithète Épiphanès qui figure aussi sur les monnaies, apparait dans le nom des prêtres éponymes d'Alexandrie jusqu'en $193^{15}$. On peut donc conclure que Polykratès accomplit sa dédicace dans le sanctuaire de Palaipaphos pendant la période finale de son administration à Chypre, entre 199 et 197, 
en suivant comme d'habitude les développements du protocole royal défini à Alexandrie.

\section{Cultes locaux}

Le culte royal, avec ses ramifications sociales et culturelles, n'est pas le seul protagoniste de la vie religieuse de la région de la Paphos hellénistique. Dans la section XIII - Inscriptions relatives à des cultes variés, on signale la mention d'une épiclèse unique d'Apollon, Myrtatès ( $\mathrm{n}^{\circ} 263$, III ${ }^{\mathrm{e}} \mathrm{s}$. av. J.-C.), ainsi que la nouvelle édition du fragment d'un règlement sacré de la même époque, concernant des punitions pour des personnes ayant contrevenu à une prescription (perdue). Le décret est préservé sur un bloc remployé dans le sanctuaire d'Agia Moni, au nord de la chōra paphienne. Le texte fait la distinction entre le type de punition destiné à une personne libre et à un esclave. Cayla associe ce document à l'attestation de la dédicace de colonnes à Héra par le roi Nikoklès sur le même site, ca. 320-310 av. J.-C. (ICS ${ }^{2}$ 90), mais l'hypothèse d'une pierre errante n'est pas à exclure. Par ailleurs, plusieurs documents font référence à une activité oraculaire dans le sanctuaire d'Aphrodite (cf. le mantiarchēs aux $\mathrm{n}^{\text {os }} 86-87$, du $\mathrm{I}^{\mathrm{er}} \mathrm{s}$. av. J.-C.; le $n^{\circ} 264$ est peut-être un fragment de réponse oraculaire métrique d'époque impériale), tandis que la mention d'un prêtre d'Aphrodite, de Zeus Polieus et d'Héra $\left(n^{\circ} 86\right)$ doit renvoyer à un culte actif à Nea Paphos au $\mathrm{I}^{\mathrm{er}} \mathrm{s}$. av. J.-C., lorsque les documents attestent une nouvelle évolution de la vie civique par rapport à la haute période hellénistique.

9 Le document $\mathrm{n}^{\circ} 81$ (fin $\mathrm{III}^{\mathrm{e}}$ - début $\mathrm{II}^{\mathrm{e}} \mathrm{s}$.) nous fait connaître Démokratès, fils de

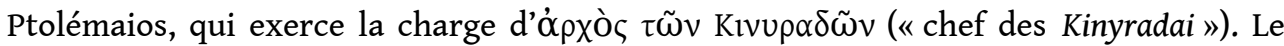
mot $\grave{\alpha} \rho x o ́ \varsigma$ est une forme dialectale attestée dans trois inscriptions syllabiques de la fin du IV $\mathrm{e}$. av. J.-C., dont deux apparaissent dans les inscriptions du sanctuaire hypogée d'Apollon Hylatès près de Nea Paphos. Les Kinyradai sont probablement une association cultuelle revendiquant une descendance du roi mythique Kinyras, le fils d'Apollon revendiqué comme ancêtre par les derniers souverains de Paphos. À l'époque impériale, une forme de continuité avec cette association est perceptible dans le titre de Kinyarchos porté par un personnage dont un portrait fut érigé dans le sanctuaire $\left(n^{\circ} 166, \mathrm{I}^{\mathrm{er}}-\mathrm{II}^{\mathrm{e}} \mathrm{s}\right.$. ap. J.-C.). La base des statues d'un couple ayant fondé un sanctuaire de Tychè et y exerçant la charge de prêtres à vie de la Tychè de Paphos $\left(\mathrm{n}^{\circ} 167\right.$, début III ${ }^{\mathrm{e}} \mathrm{s}$. ap. J.-C.) date aussi de la période impériale. Enfin, on compte parmi les témoignages de la vie religieuse locale le dossier du village d'Armagetti, au nord de Paphos. Ce site a préservé plusieurs témoignages du culte d'Opaôn Mélanthios, divinité épichorique parfois associée à Apollon et dont on soupçonne un lien avec le passage des jeunes hommes à l'âge adulte ( $\mathrm{n}^{\text {os }} 242-261$; environ $\mathrm{I}^{\text {er }}$ s. av. J.-C. $-\mathrm{II}^{\mathrm{e}} / \mathrm{III}{ }^{\mathrm{e}}$ s. ap. J.-C.).

\section{Les honneurs pour les empereurs romains}

On conclura sur quelques considérations à propos du culte et des honneurs pour les empereurs romains dans la région de Paphos. Comme l'observe Cayla, la documentation montre un déplacement progressif des lieux et des stratégies du culte impérial depuis le sanctuaire d'Aphrodite à Palaipaphos vers la ville Nea Paphos, entre le $\mathrm{I}^{\mathrm{er}}$ et le $\mathrm{II}^{\mathrm{e}} \mathrm{s}$. ap. J.-C. Sous la dynastie julio-claudienne, le site de Palaipaphos demeure un lieu 
d'importance capitale pour les initiatives des élites de la ville désireuses d'ériger des statues et de déployer des dédicaces honorant les membres de la famille impériale. Le sanctuaire a aussi préservé un bloc de marbre portant la longue formule d'un serment pour plusieurs divinités ainsi que pour Tibère, célébré comme descendant d'Aphrodite $\left(n^{\circ} 108\right)$. Dans une analyse détaillée du document, Cayla met en exergue la construction à plusieurs niveaux de l'identité de Paphos par le biais des renvois cultuels et mythiques : ainsi, la mention locale d'Aphrodite (accompagnée ici de l'épiclèse Akraia) et d'un Apollon renvoyant à la tradition du fondateur Kinyras (selon la nouvelle lecture

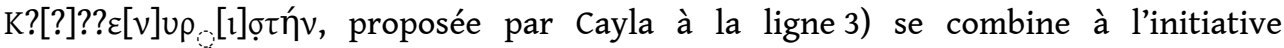
régionale du Koinon de Chypre et à la référence à Aphrodite en tant que déesse dynastique de la famille impériale. Déjà sous la première dynastie, d'ailleurs, on constate un rôle croissant de Nea Paphos dans les honneurs octroyés par la cité aux souverains romains : une attention particulière pour Livie, attestée à Palaipaphos par deux inscriptions la mentionnant probablement comme nouvelle Aphrodite (ou Déméter ?; $\left.\mathrm{n}^{\mathrm{os}} 104,112\right)$, trouve, selon Cayla (p.170-171), son pendant à Néa Paphos dans le toponyme de l'église de la Panagia Theoskepasti, qui recèlerait la dénomination Thea Sebastè portée par la femme d'Auguste ${ }^{16}$. On connait aussi deux concours organisés en l'honneur de membres de la famille impériale, qui doivent avoir eu lieu dans des structures situées dans la cité et non près du vieux sanctuaire $\left(n^{\circ} 156\right.$, fête pour Germanicus; $n^{\circ} 157$, Nérôneia). Enfin, le théâtre érigé à l'époque impériale sur le site de Fabrika est dédié à Zeus Kapitolios et aux empereurs Antonin et Marc Aurèle $\left(\mathrm{n}^{\circ} 120\right)$. Le dossier concernant les honneurs pour les empereurs se complète par le nom de quelques grands-prêtres d'empereurs de la dynastie julio-claudienne ( ${ }^{\circ \mathrm{os}} 155-157$, 164, et peut-être aussi 112) et par des mois du calendrier appelés d'après des noms impériaux, notamment Kaisarios (introduit sous Auguste) et Tibereios Sebastos ( ${ }^{\text {os }} 106$, 290)

\section{Considérations conclusives}

L'étude de Cayla est une nouveauté importante et particulièrement bienvenue dans le domaine des études de l'épigraphie de Chypre, d'autant plus que les inscriptions de l'île n'ont pas encore reçu une édition globale embrassant tous les sites et les époques antiques. Par rapport à ce desideratum, Cayla a sans doute le mérite d'avoir recueilli une documentation souvent dispersée et d'avoir ainsi offert un outil pour l'avancement de la recherche sur l'histoire de Paphos et de Chypre, ainsi que sur leurs relations avec la Méditerranée orientale hellénistique et impériale. On peut être certain que ce volume permettra de relancer plusieurs discussions sur des thématiques précises. De plus, l'analyse détaillée des formules de dédicace à Aphrodite et de la paléographie paphienne menée par Cayla promet de se révéler particulièrement utile aussi pour des études concernant d'autres cités et sites de Chypre. Cependant, les conclusions apportées par Cayla ne sont pas toujours également persuasives, surtout à cause des discordances de mise à jour de la bibliographie utilisée par l'auteur pour discuter des problèmes précis. En ce qui concerne la vie religieuse de Paphos, on constate une tendance à tirer des documents des conclusions hypothétiques, voire risquées, concernant la topographie des cultes. Deux exemples serviront à clarifier cette affirmation. Dans la discussion de l'identification, séduisante, du site de l'église de la Panagia Theoskepasti avec un lieu de culte de Livie Thea Sebastè, Cayla (p. 170-171) 
ajoute que l'église a livré, en situation de remploi, la base de la statue d'un prêtre d'une Cléopâtre $\left(\mathrm{n}^{0} 44\right)^{17}$. De cette observation, l'A. tire la conclusion que ce site aurait abrité un lieu de culte royal lagide avant d'être réinvesti pour la femme d'Auguste, et que «avant cela, Arsinoé Philadelphe y a peut-être eu son sanctuaire» (p.72). Malheureusement, rien ne permet de confirmer la thèse concernant l'existence d'un lieu de culte d'une Cléopâtre, parce que la base en question pourrait être arrivée sur le site bien plus tard, à l'époque byzantine, et même provenir d'un lieu différent. La mention d'un sanctuaire d'Arsinoé II est, quant à elle, entièrement spéculative. Le deuxième texte à discuter $\left(\mathrm{n}^{\circ} 75\right)$ est un décret honorifique rédigé $\mathrm{au} \mathrm{II}^{\mathrm{e}} \mathrm{s}$. av J.-C. par une association de soldats en faveur d'un ingénieur militaire lycien. Le document atteste l'institution d'un jour éponyme, qui se veut éternel, en l'honneur de la personne concernée, en faveur de laquelle les soldats sacrifieront dans deux contextes différents correspondant à deux sous-sections de l'association : les membres anciens sacrifieront à Aphrodite à Palaipaphos, tandis que les jeunes feront un sacrifice à Létô, à Nea Paphos. Ce document est le seul du corpus de Paphos mentionnant un culte de Létô, sûrement importé par les membres lyciens de la garnison. Or, contrairement à ce que suggère Cayla (p. 199), cela ne suffit pas à postuler l'existence d'un sanctuaire de cette déesse, fréquenté par les soldats en garnison à Paphos. Le fait que le sacrifice à Létô soit accompli par le groupe des jeunes de l'association de militaires suggère que cette offrande pourrait plutôt avoir eu lieu dans le gymnase, qui se trouvait à Nea Paphos (bien que sa situation exacte demeure inconnue) et qui était certainement fréquenté par les mercenaires lagides. Il faudra sans doute reconsidérer ces dossiers en gardant à l'esprit deux réserves méthodologiques fondamentales : s'abstenir de la reconstruction de lacunes de la documentation par analogie et rappeler que la moindre référence à un acte rituel pour une divinité n'implique pas en soi l'existence d'un sanctuaire qui lui appartienne.

\section{NOTES}

1. Jean-Baptiste CAYLA, Les Inscriptions de Paphos. La cité chypriote sous la domination lagide et à l'époque impériale, Lyon, Mom Éditions, 2018. 1 vol. $21 \times 30 \mathrm{~cm}, 565$ p. (Travaux de la Maison de l'Orient et de la Méditerranée, 74). ISBN : 978-2-35668-056-3.

2. S.G. CANEVA, « L'importance de la matérialité. Le rôle des petits autels, plaques et bases inscrits dans la compréhension des cultes pour les souverains ", in S.G. CANEVA (éd.), The Materiality of Hellenistic Ruler Cults, Liège, 2020, p. 21-64 (Kernos Suppl., 36).

3. Par rapport à l'édition du texte par T.B. MITFORD, «The Hellenistic Inscriptions of Old Paphos », ABSA 56 (1961), p. 33, $\mathrm{n}^{\circ} 88$, Cayla (p.142) interprète de manière convaincante la mention d'Aphrodite dans la partie préservée de la dernière ligne non pas comme un datif (dédicace), mais comme un génitif, et complète cette ligne et la lacune dans la deuxième moitié de la ligne précédente avec le titre de la prêtrise d'Hélénos. Cette restitution se justifie par comparaison avec le même titre porté par ce personnage dans Salamine XIII 81.

4. Cayla (p. 170) lui attribue cette fonction, tandis que les savants précédents l'assignaient à sa fille Artémo. Voir par ex. OGIS 159; récemment, cf. A. BIELMAN SANCHEZ, G.LENZO, Inventer le 
pouvoir au féminin : Cléopâtre I et Cléopâtre II, reines d'Égypte au II $^{\mathrm{e}}$ s. av. J.-C., Berne, 2015, p. 409-411. La nouvelle interprétation de Cayla se justifie en considération du fait que la partie supérieure du texte cite le titre aulique et les autres fonctions exercées par Séleukos. Ce sont bien celles-ci, et non pas les possibles charges d'Artémo, qui justifient l'initiative des soldats qui ont dédié cette statue à la fille d'un personnage qu'ils voulaient honorer, selon une logique d'honneurs familiaux bien connue à la basse époque hellénistique. De plus, l'attribution de la prêtrise chypriote à Séleukos plutôt qu'à sa fille résout le problème posé par le fait qu'Artémo revêtait déjà la prêtrise d'Arsinoé III à Alexandrie pendant les mêmes années.

5. La question de l'identité de cette Cléopâtre demeure sans solution, car la lacune après le nom de la reine à la ligne 4 est trop courte pour y ajouter n'importe quelle épiclèse permettant de l'identifier. Si cette évaluation de la longueur de la lacune est correcte, ce manque de précision par rapport à la titulature et donc à l'identité de la reine honorée est difficilement explicable : cf. BIELMAN SÁNCHEZ, LENZO (2015), p. 410.

6. On est tenté, avec Cayla, de voir le même mécanisme en action dans la dédicace fragmentaire $\mathrm{n}^{\circ} 88$, où le sanctuaire fondé par la personne honorée pourrait être encore une fois celui d'un Ptolémée.

7. Un terme de comparaison est offert par la base d'une statue de Marc Antoine érigée à

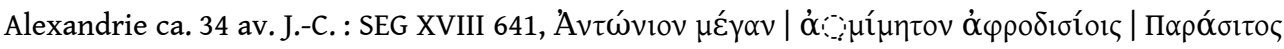

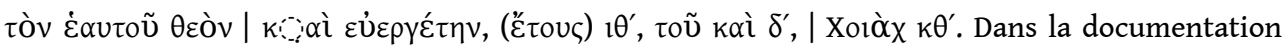
épistolaire entre les administrateurs de la période augustéenne, on compte plusieurs attestations de cet usage dans l'archive d'Athénodôros, actif dans le nome Héracléopolite : BGU XVI 2600 (13

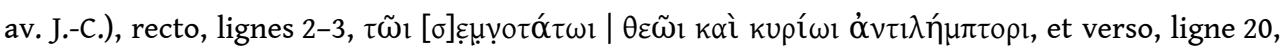

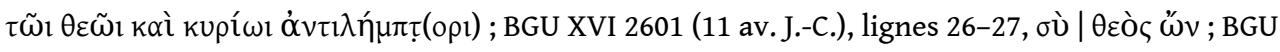

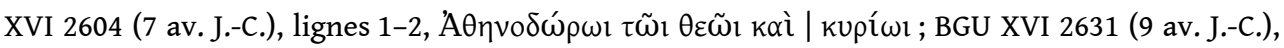

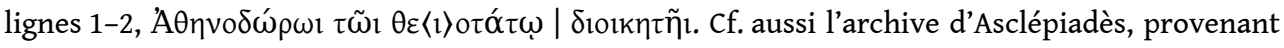

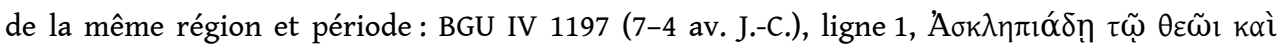

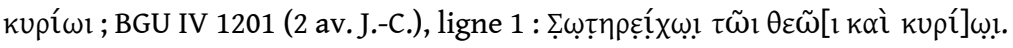

8. Voir aussi, avec plus de détails, J.-B. CAYLA, « Antoine, Cléopâtre, et les technites dionysiaques à Chypre », BCH 141-1 (2017), p. 313-336.

9. L. ROBERT, «Sur un décret d'Ilion et un papyrus concernant des cultes royaux », in A.E. SAMUEL

(éd.), Essays in Honor of C.B. Welles, New Haven, 1966, p. 175-210 [= OMS, VII, p. 599-635].

10. Cf. ci-dessus, n. 2 .

11. Pour les raisons qui invitent à préférer l'hypothèse d'un culte posthume, voir S.G. CANEVA, From Alexander to the Theoi Adelphoi: Foundation and Legitimation of a Dynasty, Leuven, 2016, p. $129-178$.

12. I. MŁYNIARCZYK, Nea Paphos in the Hellenistic Period, Varsovie, 1990, p.115-118;

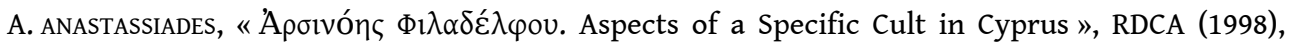
p. 129-140.

13. T.B. MITFORD, «Contributions to the Epigraphy of Cyprus: Some Hellenistic Inscriptions », APF 13 (1938), p. 28, $n^{\circ} 13$. Pour la kanephoros du document d'Idalion, cf. CSI I 93 (= I.Kition T $82 ; 254$ av. J.-C.).

14. Voir à ce propos les cas analysés dans S.G. CANEVA, L. BRICAULT, «Sarapis, Isis et la continuité dynastique lagide. À propos de deux dédicaces ptolémaïques d'Halicarnasse (RICIS 305/1702) et de Kaunos (RICIS Suppl. II 305/2002) », Chiron 49 (2019), p. 1-22 ; S.G. CANEVA, « Ptolemy II, Son of Ptolemy Soter, and the Ideology of Salvation: From Civic Acclamation to Dynastic Title », ZPE 214 (2020), p. 133-150.

15. E. LANCIERS, «The Development of the Greek Dynastic Cult under Ptolemy V », APF 60-2 (2014), p. 373-383, en particulier p. 374-380. 
16. Voir, avec plus de détail, J.-B. CAYLA, «Y-a-t-il un temple d'Aphrodite Paphienne à Nea Paphos? Une nouvelle hypothèse à propos du culte de la déesse de la mer à Paphos", in Cl. BALANDIER (éd.), Nea Paphos. Fondation et développement urbanistique d'une ville chypriote de l'antiquité à nos jours. Études archéologiques, historiques et patrimoniales, Bordeaux, 2016, p. 275-285, en particulier p. 282-283.

17. Cf. n. 4 .

\section{AUTEURS}

\section{STEFANO CANEVA}

Università di Padova

stefano.caneva@unipd.it 